\title{
The pre-main-sequence star V1184 Tauri (CB 34V) at the end of prolonged eclipse ${ }^{\star}$ (Research Note)
}

\author{
E. H. Semkov ${ }^{1}$, S. P. Peneva ${ }^{1}$, and S. I. Ibryamov ${ }^{1,2}$ \\ 1 Institute of Astronomy and National Astronomical Observatory, Bulgarian Academy of Sciences, 72 Tsarigradsko Shose Blvd., \\ 1784 Sofia, Bulgaria \\ e-mail: esemkov@astro.bas.bg \\ 2 Department of Theoretical and Applied Physics, Faculty of Natural Sciences, University of Shumen, 115 Universitetska Str., \\ 9712 Shumen, Bulgaria
}

Received 13 July 2015 / Accepted 1 September 2015

\begin{abstract}
Aims. V1184 Tau (CB 34V) lies in the field of the Bok globule CB 34 and was discovered as a large amplitude variable in 1993. According to the first hypothesis of the variability of the star, it is a FU Orionis candidate erupted between 1951 and 1993 . During subsequent observations, the star manifests large amplitude variability interpreted as obscuration from circumstellar clouds of dust. We included V1184 Tau (CB 34V) in our target list of highly variable pre-main-sequence stars to determine the reasons for the variations in the brightness of this object.

Methods. Data from BVRI photometric observations of the young stellar object V1184 Tau, obtained in the period 2008-2015, are presented in the paper. These data are a continuation of our optical photometric monitoring of the star began in 2000 and continuing to date. The photometric observations of V1184 Tau were performed in two observatories with two medium-sized and two small telescopes.

Results. Our results indicate that during periods of maximum light the star shows characteristics typical of T Tauri stars. During the observed deep minimum in brightness, however, V1184 Tau is rather similar to UX Orionis objects. The deep drop in brightness began in 2003 ended in 2015 as the star has returned to maximum light. The light curve during the drop is obviously asymmetric as the decrease in brightness lasts two times longer than the rise. The observed colour reverse on the colour-magnitude diagrams is also confirmation of obscuration from circumstellar clouds of dust as a reason for the large amplitude variability in the brightness.
\end{abstract}

Key words. stars: pre-main sequence - stars: variables: T Tauri, Herbig Ae/Be - stars: individual: V1184 Tau

\section{Introduction}

Photometric variability is a fundamental characteristic of all types of pre-main-sequence (PMS) stars. In many cases, the discovery of variability is a proof for the membership of stars in a certain group of young objects. Depending on the mass, PMS stars are divided in two main groups: the widely distributed T Tauri stars (TTS), determined as young low-mass stars $\left(M \leq 2 M_{\odot}\right)$ with emission line spectra and irregular photometric variability; and the more massive $\left(M>2 M_{\odot}\right)$ Herbig Ae/Be stars (HAEBE). Respectively TTS can be divided in two subgroups: classical T Tauri (CTT) stars surrounded by massive accretion circumstellar disks and weak line T Tauri (WTT) stars without indications of accretion from the disk (Bertout 1989).

In accordance with Herbst et al. (2007) the photometric variability of WTT stars is caused by the presence of large cool spots or groups of spots analogous to sunspots. The periods of variability on timescales of days and amplitudes up to $0.6(I)$ are typical of the WTT stars variability. The brightness variations of CTT stars are more complicated: the variability is produced by mixture of cool and hot surface spots with non-periodic variations and amplitudes up to 2-3 mag (I).

\footnotetext{
$\star$ Appendix $\mathrm{A}$ is available in electronic form at http://www. aanda.org
}

The very rare phenomena in PMS evolution, i.e. large amplitude, long lasting eruptions, are grouped into two main types: FU Orionis (FUor) and EX Lupi. The outburst of FUor objects usually continues over several decades, and the time of the rise of brightness is less than the time of the decline (see Audard et al. 2014; Reipurth \& Aspin 2010, and references therein). Well-studied FUors have the following same characteristics: a $\Delta V \approx 4-6$ mag. amplitude of the outburst, connection with reflection nebulae, belonging to regions of star formation, an F-G supergiant spectrum in the optical range, a P Cyg profile of $\mathrm{H} \alpha$ line and $\mathrm{Na}$ I doublet, a strong LiI $6707 \AA$ absorption line, and CO bands in near-infrared spectra (Herbig 1977).

A sizable number of HAEBE stars with spectral type later than A0 and some early F-G types TTS exhibit very strong photometric variability with abrupt quasi-Algol drops in brightness with amplitudes reaching to 3 mag. ( $V)$ (Nata et al. 1997). During the very deep minima in brightness an increasing of polarization and peculiar colour variability are observed. The prototype of this group of PMS stars named UXors is UX Orionis. The widely accepted explanation of its variability is a variable extinction from orbiting circumstellar clumps or clouds of dust or from edge-on circumstellar disks (Grinin et al. 1991).

The unique PMS star V1184 Tau (also known as CB 34V) discovered by Yun et al. (1997) is located in the field of the Bok 


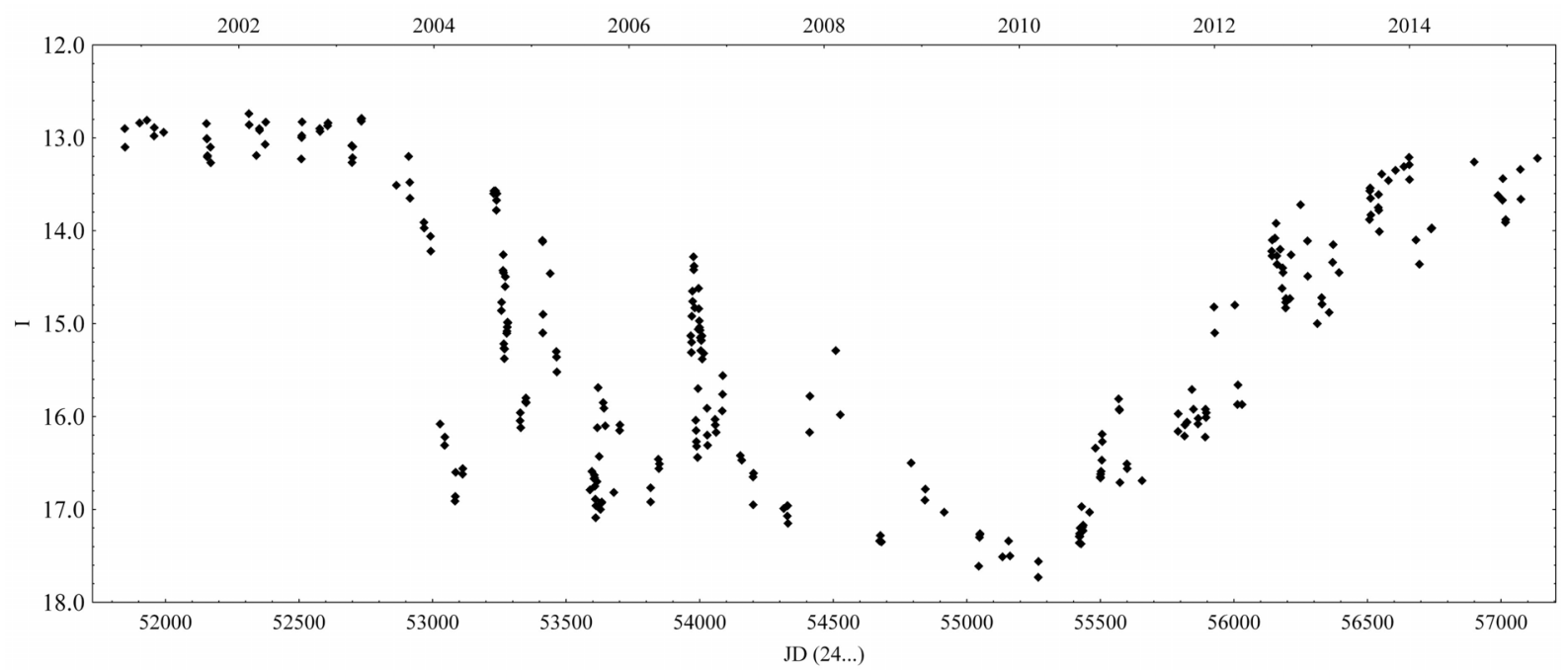

Fig. 1. I light curve of V1184 Tau for the period October 2000-April 2015.

globule CB 34 (Clemens \& Barvainis 1988). The comparison of CCD images that Yun et al. (1997) obtained in 1993 with plates from Palomar Observatory Sky Survey obtained in 1951 reveals an increase in brightness of this star at $3.7(R)$. The first supposition of Yun et al. (1997) about the nature of V1184 Tau is that it exhibits a FUor type of outburst in optical wavelengths. Alves et al. (1996) defined the spectral type of V1184 Tau as G5 (III-IV), the mass of the star $\sim 2 M_{\odot}$, and the age $\sim 10^{6} \mathrm{yrs}$. Tackett et al. (2003) discovered a 2.372 days rotation period of V1184 Tau, suggesting the presence of large cool spots on the stellar surface.

The data presented in this paper are a continuation of the optical photometric monitoring of V1184 Tau began in 2000. The photometric and spectroscopic study presented in our first paper (Semkov 2003) reveals V1184 Tau as a possible WTT star with an amplitude of $0.5(I)$ and spectral variability. In our second paper (Semkov 2004), the beginning of a new very deep minimum in brightness of V1184 Tau was reported. In the third paper from our study (Semkov 2006), new data from optical photometry and spectroscopy of V1184 Tau in the period during the deep minimum are reported. In Semkov et al. (2008), data from archival photographic plates are presented, which proves that an unknown minimum of brightness exists during the approximate period 1980-1985. Optical photometric data obtained at the time of our photometric monitoring have been published in Tackett et al. (2003), Barsunova et al. (2006), and Grinin et al. (2009).

\section{Observations}

The photometric observations of V1184 Tau were performed in two observatories with four telescopes: the 2-m RitcheyChrétien-Coudé, the 50/70-cm Schmidt and 60-cm Cassegrain telescopes of the Rozhen National Astronomical Observatory (Bulgaria), and the 1.3-m Ritchey-Chrétien telescope of the Skinakas Observatory ${ }^{1}$ of the University of Crete (Greece).

The observations were performed with five types of CCD cameras: Vers Array 1300B at the 2-m RCC telescope, ANDOR DZ436-BV at the 1.3-m RC telescope, SBIG STL-11000M and FLI PL16803 at the 50/70-cm Schmidt telescope, and FLI

\footnotetext{
1 Skinakas Observatory is a collaborative project of the University of Crete, the Foundation for Research and Technology, Greece, and the Max-Planck-Institut für Extraterrestrische Physik, Germany.
}

PL9000 at the $60-\mathrm{cm}$ Cassegrain telescope. The technical parameters for the CCD cameras used, observational procedure, and data reduction process are described in Ibryamov et al. (2015). All frames were taken through a standard Johnson-Cousins set of filters. To minimize the light from the surrounding nebula all data were analysed using the same aperture, which was chosen as $4^{\prime \prime}$ radius. The background is taken from $15^{\prime \prime}$ to $20^{\prime \prime}$. As a reference the $I R V B$ comparison sequence reported in Semkov (2003, 2006) was used.

\section{Results and discussion}

The new data from our optical photometric observations of V1184 Tau are presented in Table A.1. The columns contains the date and Julian date (J.D.) of observation, $I R V B$ magnitudes of V1184 Tau, the telescope and CCD camera used. The mean values of instrumental errors of our photometric study are listed in Semkov et al. (2008). In very deep minimums the star can be observed only in $V, R$, and $I$ bands with the middle size telescopes (2-m RCC and 1.3-m RC) and only in $R$ and $I$ bands with the small telescopes (50/70-cm Schmidt and 60-cm Cassegrain). The $I$ light curve of V1184 Tau from all our observations is shown in Fig. 1. The error bars are not shown in the figure, as their size is comparable to the symbols used.

The data collected during our long-term photometric monitoring suggest that from October 2000 to April 2003 the brightness of V1184 Tau varies with an amplitude of about $0.5(I)$ without increasing or decreasing. Since August 2003 a gradual decrease in brightness has begun and the magnitude of the star decreased with $\sim 4^{\mathrm{m}}$ (I) until March 2004. Over the next six years, the brightness of the star changed rapidly, and several rises and drops with amplitudes of several magnitudes are observed. The minimum brightness of V1184 Tau $(I=17.73)$ was registered in March 2010. Therefore, the process of decrease in brightness continues nearly seven years.

Since the summer of 2010 the brightness of the star began to rise gradually until the spring of 2015 when it reached values close to the maximum light. Process of increasing in brightness is relatively fast and with a relatively smaller brightness variations, unlike the process of decreasing in brightness. Therefore, the deep minimum in brightness of V1184 Tau lasts around 12 years and the registered amplitude is $\Delta I \approx 4 \mathrm{~m}$. 8 . 
E. H. Semkov et al.: The pre-main-sequence star V1184 Tauri (CB 34V) at the end of prolonged eclipse $(R N)$

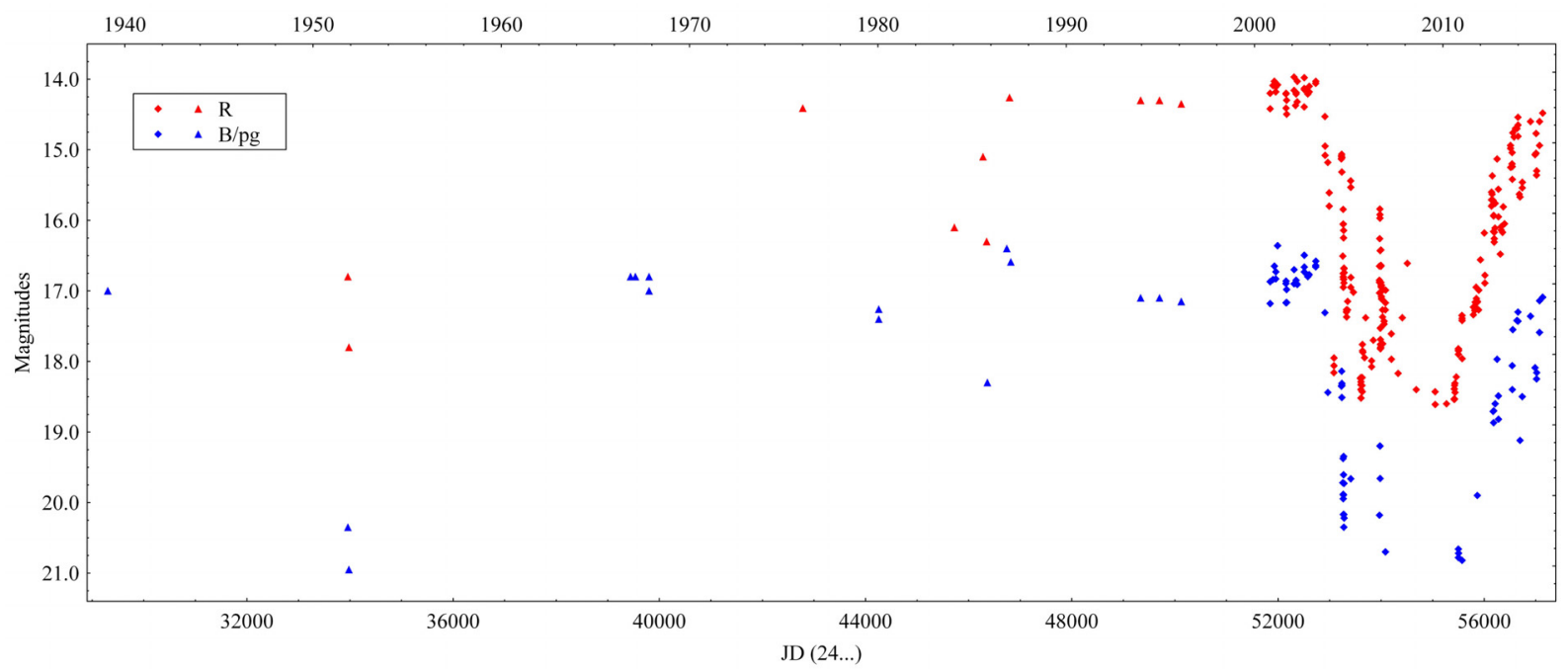

Fig. 2. Historical $R$ and $B / p g$ light curves of V1184 Tau.
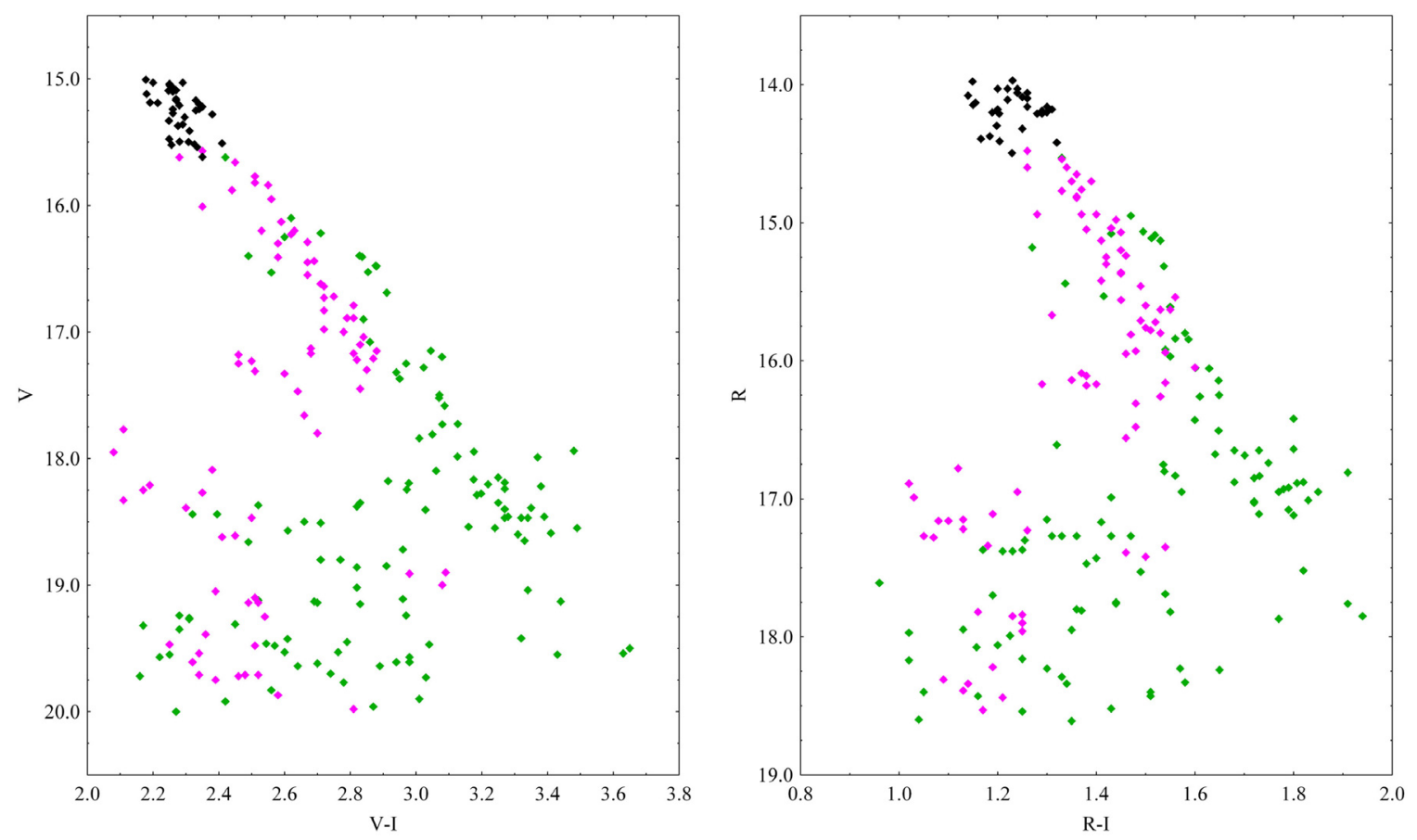

Fig. 3. $V / V-I$ and $R / R-I$ diagrams for the period of our photometric observations. In the figure, the black diamonds denote observations from October 2000 to April 2003 (maximum level of brightness out of obscuration), the green diamonds observations from August 2003 to March 2010 (when the brightness of the star dropped) and the pink diamonds from August 2010 to April 2015 (when the brightness of the star increased).

Figure 2 shows the long-term $B / p g$ and $R$ light curves of V1184 Tau from all available observations. The diamonds denote our CCD photometric data; triangles denote the photographic data collected from archives of several telescopes (Semkov et al. 2008). The figure shows that a total of three deep minimum in brightness have been registered to date: the first around 1951, the second between 1976 to 1985, and the last from 2003 to 2015 . Duration of the first minimum documented on the photographic plates from Palomar Observatory Sky Survey is still undetermined, while the duration of the second minimum ranges between six and nine years. Detailed photometric study of the third minimum indicates a complex asymmetrical light curve, which is evidence of the irregular structure of the eclipsing object.
The multi-colour photometry obtained during the deep minimum allows us to examine the variation of colour indices with stellar brightness. In Fig. 3 we plot the measured colour indices $V-I$ versus $V$ and $R-I$ versus $R$ stellar magnitude. The results from our study indicate that the star is not obscured by a compact object, but rather from large and dense clouds of dust. Also, our data indicate that the star has been relatively redder during the decrease in brightness than during the rise in brightness.

During the maximum light the colour variability (star becomes redder as it fades) is typical for WTT stars with large cool spots. This variability is caused by the rotation of the spotted surface. During the beginning of the eclipse the star also becomes redder, when its light is covered by dust clouds on the line of seeing. In this case, we assume that the reddening of the star is 
caused by the variable extinction from the circumstellar environment. However, from a certain magnitude called turning point (in this case $V \sim 18 \mathrm{mag}$ ) the colour of the star becomes bluer. The reason for this change is that the obscuration rise vastly and the part of the scattered light in the total observed light from the star is significant.

This effect of colour reversal (so-called "blueing") was described in many papers (Bibo \& The 1990; Grinin et al. 1994). In accordance with the model of dust clumps obscuration, the observed colour reversal is produced by the scattered light from the small dust grains. Our photometric data confirm the existence of a blueing effect in the colour/magnitude diagrams of V1184 Tau, a typical feature of the PMS stars from UXor type. This is independent evidence that the variability of V1184 Tau is dominated by the variable extinction from circumstellar environment. Meanwhile, large cool spots should continue to form on the stellar surface, which additionally modify the brightness and the colour of the star. Therefore, we observe a combination of both types of variability, which contributes to the large deviation of the points on the colour/magnitude diagrams. The main cause of the observed large deviation of the points, however, should be the strong fluctuations of the scattered light during our long-term photometric monitoring of the extinction event.

Based on data from infrared $J H K$ photometry, Grinin et al. (2009) propose that the increase in extinction and the drop in star's brightness can be induced by enhanced accretion from the circumstellar disks. The lack of a fall in the $K$ band is explained by a disk model with a puffed up inner rim, whose effective width is increased as a result of enhanced accretion rate. The presence of another reason for stellar variability, i.e. episodic accretion, may also increase the dispersion of points in the $V / V-I$ and $R / R-I$ diagrams.

The results from our 15 years of photometric monitoring show that V1184 Tau has no analogue among the PMS variables. A similar amplitude and duration of the decrease in brightness was only observed in the case of CQ Tau (Grinin et al. 2008). Unlike V1184 Tau, which is WTT star, CQ Tau is a typical HAEBE star with a bigger mass and from an earlier spectral type. The observed decrease in brightness during the period 2003-2015 lasted somewhat longer than our prediction
(Semkov et al. 2008), which still does not exclude the presence of the periodicity. The extremely large amplitude, rapid variability in brightness and significant deviation of colour indices can only be explained by the presence of several variability mechanisms, with variable extinction the dominant mechanism.

Acknowledgements. The authors thank the Director of Skinakas Observatory Prof. I. Papamastorakis and Prof. I. Papadakis for the award of telescope time. This research has made use of the NASA's Astrophysics Data System Abstract Service, the SIMBAD database, and the VizieR catalogue access tool, operated at CDS, Strasbourg, France.

\section{References}

Alves, J. F., Hartman, L., Briceno, C., \& Lada, C. J. 1997, AJ, 113, 1395

Audard, M., Abraham, P., Dunham, M. M., et al. 2014, in Protostars and Planets VI, eds. H. Beuther, R. S. Klessen, C. P. Dullemond, \& T. Henning (Tucson: University of Arizona Press), 387

Barsunova, O. Yu., Grinin, V. P., \& Sergeev, S. G. 2006, Astron. Lett., 32, 924

Bertout, C. 1989, ARA\&A, 27, 351

Bibo, E. A., \& The, P. S. 1990, A\&A, 236, 155

Clemens, D. P., \& Barvainis, R. E. 1988, ApJS, 68, 257

Grinin, V. P., Kiselev, N. N., Minikulov, N. Kh., Chernova, G. P., \& Voshchinnikov, N. V. 1991, Ap\&SS, 186, 283

Grinin, V. P., Thé, P. S., de Winter, D., et al. 1994, A\&A, 292, 165

Grinin, V. P., Barsunova, O. Y., Shugarov, S. Y., Kroll, P., \& Sergeev, S. G. 2008, Astrophysics, 51, 1

Grinin, V. P., Arkharov, A. A., Barsunova, O. Yu., Sergeev, S. G. \& Tambovtseva, L. V. 2009, Astron. Lett., 35, 114

Herbig, G. H. 1977, ApJ, 217, 693

Herbst, W., Eislöffel, J., Mundt, R., \& Scholz, A. 2007, in Protostars and Planets V, eds. B. Reipurth, D. Jewitt, \& K. Keil (Tucson: University of Arizona Press), 297

Ibryamov, S. I., Semkov, E. H., \& Peneva, S. P. 2015, PASA, 32, e021

Natta, A., Grinin, V. P., Mannings, V., \& Ungerechts, H. 1997, ApJ, 491, 885

Reipurth, B., \& Aspin, C. 2010, in Evolution of Cosmic Objects through their Physical Activity, Yerevan, eds. H. A. Harutyunian, A. M. Mickaelian, \& Y. Terzian (Gitutyun), 19

Semkov, E. H. 2003, A\&A, 404, 655

Semkov, E. H. 2004, A\&A, 419, L59

Semkov, E. H. 2006, Astron. Nachr., 327, 328

Semkov, E. H., Tsvetkov, M. K., Borisova, A. P., et al. 2008, A\&A, 483, 537

Tackett, S., Herbst, W., \& Williams, E. 2003, AJ, 126, 348

Yun J. L., Moreira M. C., Alves J. F., \& Storm J. 1997, A\&A, 320, 167

Pages 5 to 6 are available in the electronic edition of the journal at http: //www . aanda. org 


\section{Appendix A}

Table A.1. Photometric CCD observations of V1184 Tau during the period February 2008-April 2015.

\begin{tabular}{|c|c|c|c|c|c|c|c|}
\hline Date & $\mathrm{JD}(24 \ldots)$ & $I$ & $R$ & V & $B$ & Telescope & $\mathrm{CCD}$ \\
\hline 12.02 .2008 & 54509.332 & 15.29 & 16.61 & - & - & $\mathrm{Scm}$ & ST11000 \\
\hline 29.02.2008 & 54526.397 & 15.98 & - & - & - & $\mathrm{Scm}$ & ST11000 \\
\hline 26.07.2008 & 54673.602 & 17.34 & - & - & - & $1.3 \mathrm{~m}$ & ANDOR \\
\hline 29.07 .2008 & 54676.605 & 17.28 & - & - & - & $1.3 \mathrm{~m}$ & ANDOR \\
\hline 02.08 .2008 & 54680.595 & 17.35 & 18.40 & 19.57 & - & $1.3 \mathrm{~m}$ & ANDOR \\
\hline 20.11 .2008 & 54791.491 & 16.50 & - & - & - & $\mathrm{Scm}$ & ST11000 \\
\hline 11.01.2009 & 54843.237 & 16.90 & - & - & - & $\mathrm{Scm}$ & ST11000 \\
\hline 12.01 .2009 & 54844.310 & 16.78 & - & - & - & $\mathrm{Scm}$ & ST11000 \\
\hline 24.03 .2009 & 54915.303 & 17.03 & - & - & - & $\mathrm{Scm}$ & ST11000 \\
\hline 01.08 .2009 & 55044.604 & 17.61 & - & - & - & $1.3 \mathrm{~m}$ & ANDOR \\
\hline 04.08.2009 & 55047.604 & 17.30 & - & 19.55 & - & $1.3 \mathrm{~m}$ & ANDOR \\
\hline 05.08.2009 & 55048.607 & 17.27 & 18.43 & 19.83 & - & $1.3 \mathrm{~m}$ & ANDOR \\
\hline 06.08.2009 & 55049.589 & 17.26 & 18.61 & - & - & $1.3 \mathrm{~m}$ & ANDOR \\
\hline 28.10.2009 & 55133.481 & 17.51 & - & - & - & $\mathrm{Scm}$ & FLI \\
\hline 20.11 .2009 & 55156.495 & 17.34 & - & - & - & $\mathrm{Scm}$ & FLI \\
\hline 26.11 .2009 & 55161.520 & 17.50 & - & 19.92 & - & $2 \mathrm{~m}$ & VA \\
\hline 11.03 .2010 & 55267.299 & 17.73 & - & 20.00 & - & $2 \mathrm{~m}$ & VA \\
\hline 12.03 .2010 & 55268.269 & 17.56 & 18.60 & 19.72 & - & $2 \mathrm{~m}$ & VA \\
\hline 12.08 .2010 & 55420.600 & 17.29 & 18.54 & 19.87 & - & $1.3 \mathrm{~m}$ & ANDOR \\
\hline 13.08 .2010 & 55421.603 & 17.36 & 18.53 & 19.75 & - & $1.3 \mathrm{~m}$ & ANDOR \\
\hline 14.08 .2010 & 55422.603 & 17.26 & 18.39 & 19.72 & - & $1.3 \mathrm{~m}$ & ANDOR \\
\hline 15.08 .2010 & 55423.616 & 17.29 & - & 19.61 & - & $1.3 \mathrm{~m}$ & ANDOR \\
\hline 16.08 .2010 & 55424.610 & 17.20 & 18.34 & 19.54 & - & $1.3 \mathrm{~m}$ & ANDOR \\
\hline 19.08 .2010 & 55427.617 & 17.37 & - & 19.71 & - & $1.3 \mathrm{~m}$ & ANDOR \\
\hline 21.08 .2010 & 55429.607 & 16.97 & - & 19.48 & - & $1.3 \mathrm{~m}$ & ANDOR \\
\hline 24.08 .2010 & 55432.559 & 17.22 & 18.31 & 19.47 & - & $1.3 \mathrm{~m}$ & ANDOR \\
\hline 25.08 .2010 & 55433.576 & 17.19 & - & 19.71 & - & $1.3 \mathrm{~m}$ & ANDOR \\
\hline 26.08.2010 & 55434.564 & 17.23 & 18.44 & 19.71 & - & $1.3 \mathrm{~m}$ & ANDOR \\
\hline 27.08.2010 & 55435.575 & 17.17 & - & 19.98 & - & $1.3 \mathrm{~m}$ & ANDOR \\
\hline 20.09.2010 & 55459.595 & 17.03 & 18.22 & 19.39 & - & $1.3 \mathrm{~m}$ & ANDOR \\
\hline 12.10 .2010 & 55481.545 & 16.34 & - & - & - & $1.3 \mathrm{~m}$ & ANDOR \\
\hline 29.10 .2010 & 55499.471 & 16.65 & 17.90 & 19.14 & - & $2 \mathrm{~m}$ & VA \\
\hline 31.10 .2010 & 55500.536 & 16.66 & 17.82 & 19.05 & 20.66 & $2 \mathrm{~m}$ & VA \\
\hline 31.10 .2010 & 55501.406 & 16.62 & 17.85 & 19.14 & 20.78 & $2 \mathrm{~m}$ & VA \\
\hline 01.11 .2010 & 55502.499 & 16.59 & 17.84 & 19.10 & 20.72 & $2 \mathrm{~m}$ & VA \\
\hline 04.11 .2010 & 55505.493 & 16.47 & - & - & - & Scm & FLI \\
\hline 05.11 .2010 & 55506.484 & 16.19 & - & - & - & $\mathrm{Scm}$ & FLI \\
\hline 07.11 .2010 & 55507.530 & 16.27 & - & - & - & Scm & FLI \\
\hline 06.01 .2011 & 55568.453 & 15.81 & 17.35 & 18.90 & - & $2 \mathrm{~m}$ & VA \\
\hline 08.01 .2011 & 55570.298 & 15.92 & 17.42 & 19.00 & 20.82 & $2 \mathrm{~m}$ & VA \\
\hline 10.01 .2011 & 55571.510 & 15.93 & 17.39 & 18.91 & - & $2 \mathrm{~m}$ & VA \\
\hline 11.01.2011 & 55573.304 & 16.71 & 17.96 & 19.25 & - & $2 \mathrm{~m}$ & VA \\
\hline 06.02 .2011 & 55599.347 & 16.51 & - & - & - & Sch & FLI \\
\hline 07.02 .2011 & 55600.358 & 16.56 & - & - & - & Sch & FLI \\
\hline 04.04 .2011 & 55656.278 & 16.69 & - & - & - & Sch & FLI \\
\hline 17.08.2011 & 55790.591 & 16.16 & 17.34 & 18.61 & - & $1.3 \mathrm{~m}$ & ANDOR \\
\hline 18.08.2011 & 55791.607 & 15.97 & 17.23 & 18.47 & - & $1.3 \mathrm{~m}$ & ANDOR \\
\hline 10.09 .2011 & 55815.494 & 16.21 & 17.28 & 18.62 & - & $1.3 \mathrm{~m}$ & ANDOR \\
\hline 12.09 .2011 & 55816.565 & 16.09 & 17.22 & 18.39 & - & $1.3 \mathrm{~m}$ & ANDOR \\
\hline 20.09.2011 & 55824.507 & 16.06 & 17.16 & - & - & $1.3 \mathrm{~m}$ & ANDOR \\
\hline 07.10 .2011 & 55842.462 & 15.71 & 16.95 & 18.09 & - & $1.3 \mathrm{~m}$ & ANDOR \\
\hline 13.10 .2011 & 55848.460 & 15.92 & 17.11 & 18.27 & - & $1.3 \mathrm{~m}$ & ANDOR \\
\hline 30.10 .2011 & 55865.463 & 16.08 & 17.16 & 18.25 & - & $2 \mathrm{~m}$ & VA \\
\hline 31.10 .2011 & 55866.468 & 16.02 & 17.15 & 18.21 & 19.90 & $2 \mathrm{~m}$ & VA \\
\hline 26.11 .2011 & 55892.430 & 16.22 & 17.27 & 18.33 & - & $2 \mathrm{~m}$ & VA \\
\hline 27.11.2011 & 55893.387 & 15.92 & - & - & - & Sch & FLI \\
\hline 29.11.2011 & 55895.456 & 16.01 & - & - & - & Sch & FLI \\
\hline 30.11 .2011 & 55896.422 & 15.96 & 16.99 & - & - & Sch & FLI \\
\hline 30.12 .2011 & 55925.532 & 14.82 & - & - & - & Sch & FLI \\
\hline 01.01 .2012 & 55928.327 & 15.10 & 16.56 & 17.80 & - & Sch & FLI \\
\hline 16.03.2012 & 56003.320 & 14.80 & 16.18 & 17.31 & - & Sch & FLI \\
\hline 26.03.2012 & 56013.322 & 15.87 & 16.89 & 17.95 & - & $2 \mathrm{~m}$ & VA \\
\hline 28.03 .2012 & 56015.326 & 15.66 & 16.78 & 17.77 & - & $2 \mathrm{~m}$ & VA \\
\hline 12.04 .2012 & 56030.279 & 15.87 & - & - & - & Sch & FLI \\
\hline
\end{tabular}


Table A.1. continued.

\begin{tabular}{|c|c|c|c|c|c|c|c|}
\hline Date & JD $(24 \ldots)$ & $I$ & $R$ & V & $B$ & Telescope & CCD \\
\hline 02.08 .2012 & 56141.602 & 14.22 & 15.71 & 17.00 & - & $1.3 \mathrm{~m}$ & ANDOR \\
\hline 03.08 .2012 & 56142.603 & 14.27 & 15.80 & 17.15 & - & $1.3 \mathrm{~m}$ & ANDOR \\
\hline 04.08 .2012 & 56143.602 & 14.10 & 15.60 & 16.89 & - & $1.3 \mathrm{~m}$ & ANDOR \\
\hline 14.08.2012 & 56153.608 & 14.08 & 15.63 & 16.89 & - & $1.3 \mathrm{~m}$ & ANDOR \\
\hline 18.08 .2012 & 56157.622 & 13.92 & 15.37 & 16.64 & - & $1.3 \mathrm{~m}$ & ANDOR \\
\hline 21.08 .2012 & 56160.572 & 14.27 & 15.78 & 17.10 & - & $1.3 \mathrm{~m}$ & ANDOR \\
\hline 22.08 .2012 & 56161.598 & 14.36 & - & - & - & Sch & FLI \\
\hline 03.09.2012 & 56173.549 & 14.20 & 15.72 & 17.04 & 18.71 & $1.3 \mathrm{~m}$ & ANDOR \\
\hline 10.09.2012 & 56180.591 & 14.62 & 16.16 & 17.45 & - & $1.3 \mathrm{~m}$ & ANDOR \\
\hline 11.09 .2012 & 56182.500 & 14.40 & 15.94 & 17.22 & 18.87 & $1.3 \mathrm{~m}$ & ANDOR \\
\hline 13.09 .2012 & 56183.541 & 14.45 & 15,93 & 17.13 & 18.70 & $1.3 \mathrm{~m}$ & ANDOR \\
\hline 23.09 .2012 & 56193.542 & 14.83 & 16.31 & 17.47 & - & $1.3 \mathrm{~m}$ & ANDOR \\
\hline 23.09 .2012 & 56193.559 & 14.77 & 16.17 & - & - & Sch & FLI \\
\hline 24.09 .2012 & 56194.531 & 14.73 & 16.26 & 17.33 & - & Sch & FLI \\
\hline 09.10 .2012 & 56209.547 & 14.73 & 16.11 & 17.23 & - & Sch & FLI \\
\hline 13.10 .2012 & 56214.396 & 14.26 & 15.76 & 16.98 & 18.60 & $2 \mathrm{~m}$ & VA \\
\hline 17.11.2012 & 56249.430 & 13.72 & 15.13 & 16.30 & 17.97 & Sch & FLI \\
\hline 14.12.2012 & 56275.562 & 14.11 & 15.56 & 16.83 & 18.49 & $2 \mathrm{~m}$ & VA \\
\hline 14.12 .2012 & 56276.414 & 14.49 & 15.95 & 17.17 & 18.82 & $2 \mathrm{~m}$ & VA \\
\hline 19.01.2013 & 56312.357 & 15.00 & 16.48 & 17.66 & - & $2 \mathrm{~m}$ & VA \\
\hline 04.02 .2013 & 56328.438 & 14.72 & 16.09 & 17.18 & - & Sch & FLI \\
\hline 05.02 .2013 & 56329.422 & 14.79 & 16.14 & 17.25 & - & Sch & FLI \\
\hline 04.03.2013 & 56356.373 & 14.88 & 16.17 & - & - & $60 \mathrm{~cm}$ & FLI \\
\hline 17.03.2013 & 56369.341 & 14.34 & 15.81 & 17.21 & - & $2 \mathrm{~m}$ & VA \\
\hline 19.03.2013 & 56371.332 & 14.15 & - & - & - & $2 \mathrm{~m}$ & VA \\
\hline 10.04 .2013 & 56393.277 & 14.45 & 16.05 & 17.30 & - & Sch & FLI \\
\hline 03.08 .2013 & 56507.572 & 13.88 & - & - & - & $2 \mathrm{~m}$ & VA \\
\hline 04.08 .2013 & 56508.573 & 13.57 & 14.94 & 16.20 & - & $2 \mathrm{~m}$ & VA \\
\hline 05.08 .2013 & 56509.575 & 13.54 & 14.98 & - & - & Sch & FLI \\
\hline 06.08 .2013 & 56510.573 & 13.54 & 14.94 & 16.13 & - & Sch & FLI \\
\hline 07.08.2013 & 56511.574 & 13.65 & - & - & - & Sch & FLI \\
\hline 08.08.2012 & 56512.571 & 13.83 & 15.25 & 16.41 & - & Sch & FLI \\
\hline 05.09 .2013 & 56540.564 & 13.75 & 15.20 & 16.44 & - & Sch & FLI \\
\hline 06.09 .2013 & 56541.546 & 13.61 & 15.04 & 16.23 & - & Sch & FLI \\
\hline 07.09 .2013 & 56542.537 & 13.78 & 15.24 & 16.45 & 18.06 & Sch & FLI \\
\hline 09.09.2013 & 56544.528 & 14.01 & 15.42 & 16.73 & 18.40 & $2 \mathrm{~m}$ & VA \\
\hline 17.09 .2013 & 56553.525 & 13.39 & 14.76 & 15.95 & 17.55 & $1.3 \mathrm{~m}$ & ANDOR \\
\hline 13.10 .2013 & 56578.592 & 13.46 & 14.82 & - & - & $60 \mathrm{~cm}$ & FLI \\
\hline 08.11 .2013 & 56604.545 & 13.35 & 14.70 & - & - & $60 \mathrm{~cm}$ & FLI \\
\hline 09.12 .2013 & 56636.332 & 13.31 & 14.70 & 15.82 & 17.42 & $2 \mathrm{~m}$ & VA \\
\hline 28.12 .2013 & 56655.432 & 13.21 & 14.54 & 15.66 & 17.30 & Sch & FLI \\
\hline 30.12 .2013 & 56656.517 & 13.29 & 14.65 & 15.84 & 17.43 & Sch & FLI \\
\hline 30.12 .2013 & 56657.279 & 13.45 & 14.81 & - & - & Sch & FLI \\
\hline 23.01 .2014 & 56681.461 & 14.10 & 15.63 & 16.89 & - & Sch & FLI \\
\hline 05.02 .2014 & 56694.412 & 14.36 & 15.67 & 17.17 & 19.12 & $2 \mathrm{~m}$ & VA \\
\hline 21.03.2014 & 56738.291 & 13.98 & 15.54 & 16.79 & 18.50 & Sch & FLI \\
\hline 23.03.2014 & 56740.302 & 13.97 & 15.46 & 16.72 & - & Sch & FLI \\
\hline 30.08 .2014 & 56899.529 & 13.26 & 14.60 & 15.77 & 17.36 & $1.3 \mathrm{~m}$ & ANDOR \\
\hline 26.11 .2014 & 56988.337 & 13.62 & 15.07 & 16.29 & 18.09 & Sch & FLI \\
\hline 14.12 .2014 & 57005.532 & 13.67 & 15.05 & 16.20 & - & Sch & FLI \\
\hline 15.12 .2014 & 57006.576 & 13.44 & 14.77 & 15.88 & - & Sch & FLI \\
\hline 25.12 .2014 & 57016.556 & 13.91 & 15.36 & 16.62 & 18.25 & $2 \mathrm{~m}$ & VA \\
\hline 25.12.2014 & 57017.346 & 13.88 & 15.30 & 16.55 & 18.16 & $2 \mathrm{~m}$ & VA \\
\hline 18.02.2015 & 57072.365 & 13.34 & 14.60 & 15.62 & 17.14 & Sch & FLI \\
\hline 20.02 .2015 & 57074.361 & 13.66 & 14.94 & 16.01 & 17.59 & Sch & FLI \\
\hline 23.04 .2015 & 57136.255 & 13.22 & 14.48 & 15.57 & 17.09 & Sch & FLI \\
\hline
\end{tabular}

